\title{
A Mixed Problem for Navier-Stokes System
}

\author{
N. Aliev, Sh. Rezapour, And M. Jahanshahi
}

\begin{abstract}
Mixed problems are changed to boundary value problems by making used of Laplace transform. In classical boundary value problems, boundary conditions are local, but boundary conditions could be global $([2,3,11])$. Every boundary value problem depends on a potential method in mathematical-physics theory. Of course, one couldn't solve some many problems by potential methods. We shall give a method in which one could reduce every boundary value problem to the second kind Fredholm integral equations and then solve it. In fact, we could obtain solution of every local, non-local or global boundary value problem by this method. Finally, we shall give some sufficient conditions for existence of solutions of the Fefferman's problem A ([4]).
\end{abstract}

\section{INTRODUCTION}

There are usually mathematical models based on differential equations, integral equations and integro-differential equations for physical and natural events. These models are frequently based on Cauchy problem, boundary value problem or mixed problem $([5-9,18-20])$. If there is the time variable in these equations, for verification of their solutions we obtain a boundary value problem which is depend on complex parameter by using the method in [15] or Laplace transform (see [7, 18]).

The boundary value problem may be in a bounded or unbounded region. We must provide boundary conditions in bounded regions, but solutions of the problems and their derivatives are periodic or tend to zero at infinity in unbounded regions.

The potential theory is useful in many boundary value problems, for example Dirichlet and Neumann problems. But, potential theory is not efficient in solving of many another problems (see [1-3, 10, 12-14, 17]). We shall give a method that it will be efficient than the potential theory.

The Euler and Navier-Stokes equations describe the motion of a fluid in $\mathbb{R}^{n}(\mathrm{n}=2$ or $\mathrm{n}=3)$. These equations are to be solved for an unknown velocity

2000 Mathematics Subject Classification. Primary: 35A99.

Key words and phrases. Navier-Stokes equations, Potential Theory, Boundary Conditions. 
vector $u(x, t)=\left(u_{i}(x, t)\right)_{1 \leq i \leq n} \in \mathbb{R}^{n}$ and pressure $p(x, t) \in \mathbb{R}$, defined for position $x \in \mathbb{R}^{n}$ and time $t \geq 0$. We restrict attention here to incompressible fluids filling all of $\mathbb{R}^{n}$. The Navier-Stokes equations are then given by

$$
\begin{aligned}
\frac{\partial u_{i}}{\partial t}+\sum_{j=1}^{n} u_{j} \frac{\partial u_{i}}{\partial x_{j}} & =\nu \Delta u_{i}-\frac{\partial p}{\partial x_{i}}+f_{i}(x, t), & & \left(x \in \mathbb{R}^{n}, t \geq 0\right) \\
\operatorname{div} u & =\sum_{i=1}^{n} \frac{\partial u_{i}}{\partial x_{i}}=0, & & \left(x \in \mathbb{R}^{n}, t \geq 0\right)
\end{aligned}
$$

with initial conditions

$$
u(x, 0)=u^{0}(x), \quad\left(x \in \mathbb{R}^{n}\right) .
$$

Here, $u^{0}(x)$ is a given $C^{\infty}$ divergence-free vector field on $\mathbb{R}^{n}, f_{i}(x, t)$ are the components of a given externally applied force (e.g. gravity), $\nu$ is a positive coefficient (the viscosity) and $\Delta=\sum_{i=1}^{n} \frac{\partial^{2}}{\partial x_{i}^{2}}$ is the Laplacian in the space variables. The Euler equations are equations (1), (2), (3) with $\nu$ is equal to zero.

Equation (1) is just Newton's low $f=m a$ for a fluid element subject to the external force $f=\left(f_{i}(x, t)\right)_{1 \leq i \leq n}$ and to the forces arising from pressure and friction. Equation (2) just says that fluid is incompressible. For physically reasonable solutions, we want to make sure $u(x, t)$ does now grow large as $|x| \longrightarrow \infty$. Hence, we will restrict to forces $f$ and initial conditions $u^{0}$ that satisfy

$$
\left|\partial_{x}^{\alpha} u^{0}(x)\right| \leq C_{\alpha K}(1+|x|)^{-K}
$$

on $\mathbb{R}^{n}$, for any $\alpha$ and $K$, and

$$
\left|\partial_{x}^{\alpha} \partial_{t}^{m} f(x, t)\right| \leq C_{\alpha m K}(1+|x|+t)^{-K}
$$

on $\mathbb{R}^{n} \times[0, \infty)$, for any $\alpha, m, K$.

We accept a solution of (1), (2) and (3) as physically reasonable only if it satisfies

$$
p, u \in C^{\infty}\left(\mathbb{R}^{n} \times[0, \infty)\right)
$$

and

$$
\int_{\mathbb{R}^{n}}|u(x, t)|^{2} \mathrm{~d} x<C, \quad \text { for all } \quad t \geq 0 \quad \text { (bounded energy). }
$$

A fundamental problem in analysis is to decide whether such smooth, physically reasonable solutions exist for the Navier-Stokes equations. To give reasonable leeway to solvers while retaining the heart of the problem, Fefferman has provided four problems A, B, C and D. The authors have verified the problems $\mathrm{C}$ and $\mathrm{D}$ in [4]. Here, we restate the problem $\mathrm{A}$.

(A) Existence and Smoothness of Navier-Stokes Solutions on $\mathbb{R}^{3}$. Take $\nu>0$ and $n=3$. Let $u^{0}(x)$ be any smooth, divergence-free vector field satisfying (4). Take $f(x, t)$ to be identically zero. Then there exist smooth 
functions $p(x, t), u_{i}(x, t)$ on $\mathbb{R}^{3} \times[0, \infty)$ that satisfy (1), (2), (3), (6) and (7).

Definition 1.1. A bounded function $f:(a, b) \rightarrow \mathbb{R}$ is called regular if $f(x)=\frac{f\left(x^{+}\right)+f\left(x^{-}\right)}{2}$ for all $x \in(a, b)$. If $I$ is a closed or half-open interval, then a bounded function $f: I \rightarrow \mathbb{R}$ is called regular whenever $h: \mathbb{R} \longrightarrow \mathbb{R}$ so is, where

$$
h(x)= \begin{cases}f(x), & x \in I \\ 0, & \text { elsewhere. }\end{cases}
$$

Let $f:(a, b) \longrightarrow \mathbb{R}$ be a bounded function and let the set $E=\{x \in$ $(a, b): f$ is not defined at $x$ or $f$ is not continuous at $x\}$ be countable. Then, there is an unique bounded regular function $g:(a, b) \longrightarrow \mathbb{R}$ such that $\left.g\right|_{(a, b) \backslash E}=f$.

We call $x=a, \frac{m}{n}$-th repeated root of $\sqrt[n]{(x-a)^{m}}=0$ as we called $x=a$, $n$-th repeated root of $(x-a)^{n}=0$. In general, we call $x=a, \alpha$-th repeated root of $(x-a)^{\alpha}=0$ for all real $\alpha>0$.

It is well known in references that, $\alpha$ is singularity of order $-\alpha$ for the function $f(x)=x^{\alpha}$, when $\alpha<0$ and

$$
\int_{-\infty}^{x} \delta(t) \mathrm{d} t= \begin{cases}1, & x>0 \\ \frac{1}{2}, & x=0 \\ 0, & x<0,\end{cases}
$$

where $\delta$ is the Dirac's function.

It be differentiated of the equation $x \delta(x)=0$ in some mathematical works (for example, [7] and [19]), but we do not accept it in this paper and we agree it while there does not exist differentiation action. We have a similar sight on the equation $|x|^{2}=x^{2}$ ( $\mathrm{x}$ is real).

As we know in potential theory,

$$
U(x-\xi, t-\tau)=\frac{\theta(t-\tau)}{(2 a \sqrt{\pi(t-\tau)})^{n}} e^{-\frac{|x-\xi|^{2}}{4 a^{2}(t-\tau)}}
$$

is a fundamental solution of the operator $\frac{\partial}{\partial t}-a^{2} \Delta_{x}$ respect to the time variable, where $\theta$ is the Heviside function, and

$$
U_{3}(x-\xi)=-\frac{1}{4 \pi|x-\xi|}
$$

is a fundamental solution of the 3-dimensional Laplacian $\Delta_{x}=\sum_{j=1}^{3} \frac{\partial^{2}}{\partial x_{j}^{2}}$ in direction of perpendicular vectors on the boundary. Now, we claim that

$$
U_{2}(x-\xi)=\frac{1}{2 \pi} \ln \sqrt{\left(x_{1}-\xi_{1}\right)^{2}+\left|x_{2}-\xi_{2}\right|^{2}}
$$


is fundamental solution of the 2-dimensional Laplace equation in direction of $x_{2}$. In fact,

$$
\begin{aligned}
\frac{\partial U_{2}(x-\xi)}{\partial x_{2}}= & \frac{1}{2 \pi} \frac{2\left|x_{2}-\xi_{2}\right| e\left(x_{2}-\xi_{2}\right)}{\left(x_{1}-\xi_{1}\right)^{2}+\left|x_{2}-\xi_{2}\right|^{2}} \\
\frac{\partial U_{2}(x-\xi)}{\partial x_{1}}= & \frac{1}{2 \pi} \frac{x_{1}-\xi_{1}}{\left(x_{1}-\xi_{1}\right)^{2}+\left|x_{2}-\xi_{2}\right|^{2}} \\
\frac{\partial^{2} U_{2}(x-\xi)}{\partial x_{2}^{2}}= & \frac{1}{\pi} \delta\left(x_{2}-\xi_{2}\right) \frac{\left|x_{2}-\xi_{2}\right|}{\left(x_{1}-\xi_{1}\right)^{2}+\left|x_{2}-\xi_{2}\right|^{2}}+ \\
& +\frac{1}{2 \pi} \frac{1}{\left(x_{1}-\xi_{1}\right)^{2}+\left|x_{2}-\xi_{2}\right|^{2}}- \\
& -\frac{1}{\pi} \frac{\left|x_{2}-\xi_{2}\right|^{2}}{\left[\left(x_{1}-\xi_{1}\right)^{2}+\left|x_{2}-\xi_{2}\right|^{2}\right]^{2}},
\end{aligned}
$$

and

$$
\frac{\partial^{2} U_{2}(x-\xi)}{\partial x_{1}^{2}}=\frac{1}{2 \pi} \frac{-\left(x_{1}-\xi_{1}\right)^{2}+\left|x_{2}-\xi_{2}\right|^{2}}{\left[\left(x_{1}-\xi_{1}\right)^{2}+\left|x_{2}-\xi_{2}\right|^{2}\right]^{2}},
$$

where $e$ is the symmetric Heaviside function. Thus, $\Delta_{2} U_{2}(x-\xi)=\delta(x-\xi)$. Also,

$$
\begin{aligned}
U_{0}(x-\xi)= & \frac{\theta\left(x_{2}-\xi_{2}\right)}{2 \pi} \int_{0}^{\infty} e^{-i \alpha_{1}\left(x_{1}-\xi_{1}\right)-\alpha_{1}\left(x_{2}-\xi_{2}\right)} \mathrm{d} \alpha_{1} \\
& -\frac{\theta\left(\xi_{2}-x_{2}\right)}{2 \pi} \int_{0}^{\infty} e^{i \alpha_{1}\left(x_{1}-\xi_{1}\right)-\alpha_{1}\left(\xi_{2}-x_{2}\right)} \mathrm{d} \alpha_{1},
\end{aligned}
$$

is fundamental solution of the Cauchy-Riemann operator $\frac{\partial}{\partial x_{2}}+i \frac{\partial}{\partial x_{1}}$, where $\theta$ is the Heviside function and $i^{2}=-1$ is the imaginary number, whereas some one give $\frac{1}{2 \pi} \frac{1}{x_{2}-\xi_{2}+i\left(x_{1}-\xi_{1}\right)}$ as fundamental solution of the CauchyRiemann equation ([18]). If $x_{2} \neq \xi_{2}$, then two above solutions are same. But, the above solution $U_{0}$ is a better solution when $x_{2}=\xi_{2}$.

Frequently, fractional differentiation of a function $f$ be found by Fourier transform ([16]), whereas this method is not true. We will use the Cauchy formula for definition of fractional differentiation of a function, as did Liouville.

\section{On the Problem A}

By using Laplace transformation on (1) and (2), we have

$$
\begin{gathered}
\int_{0}^{\infty} e^{-\lambda t} \frac{\partial u_{i}(x, t)}{\partial t} \mathrm{~d} t+\sum_{j=1}^{3} \int_{0}^{\infty} e^{-\lambda t} u_{j}(x, t) \frac{\partial u_{i}(x, t)}{\partial x_{j}} \mathrm{~d} t= \\
=\nu \Delta \int_{0}^{\infty} e^{-\lambda t} u_{i}(x, t) \mathrm{d} t-\frac{\partial}{\partial x_{i}} \int_{0}^{\infty} e^{-\lambda t} p(x, t) \mathrm{d} t
\end{gathered}
$$


for all $i=1,2,3$ and also,

$$
\sum_{i=1}^{3} \frac{\partial}{\partial x_{i}} \int_{0}^{\infty} e^{-\lambda t} u_{i}(x, t) \mathrm{d} t=0
$$

where $\lambda$ is a complex parameter and $\operatorname{Re} \lambda=c>0$.

By using integration by part, we have

$$
\int_{0}^{\infty} e^{-\lambda t} \frac{\partial u_{i}(x, t)}{\partial t} \mathrm{~d} t=-u_{i}^{0}(x)+\lambda \int_{0}^{\infty} e^{-\lambda t} u_{i}(x, t) \mathrm{d} t .
$$

Now, we show Laplace transforms of the functions $u_{i}$ and $p$ as following

$$
\begin{aligned}
\tilde{u}_{i}(x, \lambda) & =\int_{0}^{\infty} e^{-\lambda t} u_{i}(x, t) \mathrm{d} t \\
\tilde{p}(x, \lambda) & =\int_{0}^{\infty} e^{-\lambda t} p(x, t) \mathrm{d} t .
\end{aligned}
$$

Thus,

$$
\begin{aligned}
\Delta \tilde{u}_{i}(x, \lambda)-\frac{\lambda}{\nu} \tilde{u}_{i}(x, \lambda) & =g_{i}(x, \lambda), & & \left(i=1,2,3 ; x \in \mathbb{R}^{3}\right) \\
\sum_{j=1}^{3} \frac{\partial \tilde{u}_{j}(x, \lambda)}{\partial x_{j}} & =0, & & \left(x \in \mathbb{R}^{3}\right)
\end{aligned}
$$

where

$$
\begin{aligned}
& g_{i}(x, \lambda)=-\frac{1}{\nu} u_{i}^{0}(x)+ \\
& \quad+\frac{1}{\nu} \sum_{j=1}^{3} \int_{0}^{\infty} e^{-\lambda t} u_{j}(x, t) \frac{\partial u_{i}(x, t)}{\partial x_{j}} \mathrm{~d} t+\frac{1}{\nu} \frac{\partial}{\partial x_{i}} \tilde{p}(x, \lambda) .
\end{aligned}
$$

Left hand side of (10) is a Helmholts equation because left hand side coefficients of (10) are not depend on $i$. Hence by [18], its fundamental solution is

$$
U(x-\xi, \lambda)=-\frac{e^{-\sqrt{\frac{\lambda}{\nu}}|x-\xi|}}{4 \pi|x-\xi|},
$$

that is,

$$
\Delta_{x} U(x-\xi, \lambda)-\frac{\lambda}{\nu} U(x-\xi, \lambda)=\delta(x-\xi),
$$

where, $\lambda \in R_{\sigma}=\{\lambda:-\pi+\sigma \leq \arg \lambda \leq \pi-\sigma\}$ and we have fixed sufficiently small $\sigma>0$.

Note that if $\lambda \in R_{\sigma},|\lambda| \longrightarrow \infty$ and $x \neq \xi$, then $U(x-\xi, \lambda) \longrightarrow 0$. 
Now, multiple (10) by $U(x-\xi, \lambda)$ and integrate the result on $\mathbb{R}^{3}$. Then,

$$
\begin{array}{rl}
\int_{\mathbb{R}^{3}} \Delta \tilde{u}_{i}(x, \lambda) U(x-\xi, \lambda) \mathrm{d} & t-\frac{\lambda}{\nu} \int_{\mathbb{R}^{3}} \tilde{u}_{i}(x, \lambda) U(x-\xi, \lambda) \mathrm{d} x= \\
=\int_{\mathbb{R}^{3}} g i(x, \lambda) U(x-\xi, \lambda) \mathrm{d} x, \quad(i=1,2,3)
\end{array}
$$

By using Gauss-Ostrogradskii for the first integral of left hand side, from the last equation and by (7), we have

$$
\begin{aligned}
\int_{\mathbb{R}^{3}} \tilde{u}_{i}(x, \lambda)\left[\Delta_{x} U(x-\xi, \lambda)-\frac{\lambda}{\nu} U(x-\xi, \lambda)\right] \mathrm{d} x & = \\
= & \int_{\mathbb{R}^{3}} g_{i}(x, \lambda) U(x-\xi, \lambda) \mathrm{d} x .
\end{aligned}
$$

Thus by (14),

$$
\tilde{u}_{i}(\xi, \lambda)=\int_{\mathbb{R}^{3}} g_{i}(x, \lambda) U(x-\xi, \lambda) \mathrm{d} x, \quad\left(i=1,2,3 ; \xi \in \mathbb{R}^{3}\right) .
$$

By using the method in [1-3] and [10-13], we differentiate of the last equation. Then,

$$
\frac{\partial \tilde{u}_{i}(\xi, \lambda)}{\partial \xi_{k}}=\int_{\mathbb{R}^{3}} g_{i}(x, \lambda) \frac{\partial U(x-\xi, \lambda)}{\partial \xi_{k}} \mathrm{~d} t, \quad\left(i=1,2,3 ; \xi \in \mathbb{R}^{3}\right) .
$$

Now, by substituting (16) in (11), we obtain

$$
0=\sum_{i=1}^{3} \frac{\partial \tilde{u}_{i}(\xi, \lambda)}{\partial \xi_{i}}=\int_{\mathbb{R}^{3}} \sum_{i=1}^{3} g_{i}(x, \lambda) \frac{\partial U(x-\xi, \lambda)}{\partial \xi_{i}} \mathrm{~d} x
$$

Substitute (12) in recent equation. Then,

$$
\begin{aligned}
& \frac{-1}{\nu} \int_{\mathbb{R}^{3}} \sum_{i=1}^{3} u_{i}^{0}(x) \frac{\partial U(x-\xi, \lambda)}{\partial \xi_{i}} \mathrm{~d} x+ \\
&+\frac{1}{\nu} \int_{\mathbb{R}^{3}} \sum_{i, j=1}^{3} \frac{\partial U(x-\xi, \lambda)}{\partial \xi_{i}} d x \int_{0}^{\infty} e^{-\lambda t} u_{j}(x, t) \frac{\partial u_{i}(x, t)}{\partial x_{j}} \mathrm{~d} t+ \\
& \quad+\frac{1}{\nu} \int_{\mathbb{R}^{3}} \sum_{i=1}^{3} \frac{\partial \tilde{p}(x, \lambda)}{\partial x_{i}} \frac{\partial U(x-\xi, \lambda)}{\partial \xi_{i}} \mathrm{~d} x=0 .
\end{aligned}
$$


By using (14), (7) and Gauss-Ostrogradskii formula ([7]) in the last expression of recent equation, we obtain

$$
\begin{aligned}
& \frac{-1}{\nu} \int_{\mathbb{R}^{3}} \sum_{i=1}^{3} u_{i}^{0}(x) \frac{\partial U(x-\xi, \lambda)}{\partial \xi_{i}} \mathrm{~d} x+ \\
&+\frac{1}{\nu} \int_{\mathbb{R}^{3}} \sum_{i, j=1}^{3} \frac{\partial U(x-\xi, \lambda)}{\partial \xi_{i}} d x \int_{0}^{\infty} e^{-\lambda t} u_{j}(x, t) \frac{\partial u_{i}(x, t)}{\partial x_{j}} \mathrm{~d} t+ \\
& \quad+\frac{1}{\nu} \int_{\mathbb{R}^{3}} \tilde{p}(x, \lambda)\left[\delta(x-\xi)+\frac{\lambda}{\nu} U(x-\xi, \lambda)\right] \mathrm{d} x=0 .
\end{aligned}
$$

Hence by property of Dirac's function, we have

$$
\begin{aligned}
\tilde{p}(\xi, \lambda)=\frac{-\lambda}{\nu} \int_{\mathbb{R}^{3}} \tilde{p}(x, \lambda) U(x-\xi, \lambda) \mathrm{d} x+ \\
\quad+\int_{\mathbb{R}^{3}} \sum_{i=1}^{3} u_{i}^{0}(x) \frac{\partial U(x-\xi, \lambda)}{\partial \xi_{i}} \mathrm{~d} x- \\
\quad-\int_{\mathbb{R}^{3}} \sum_{i, j=1}^{3} \frac{\partial U(x-\xi, \lambda)}{\partial \xi_{i}} d x \int_{0}^{\infty} e^{-\lambda t} u_{j}(x, t) \frac{\partial u_{i}(x, t)}{\partial x_{j}} \mathrm{~d} t .
\end{aligned}
$$

Theorem 2.1. Let $\nu>0$ and $u^{0}$ satisfies (4). Then, solution of the nonlinear second type Fredholm system of equations with weak singularity (15) and (16), is a solution of (10).

Theorem 2.2. Let $\nu>0$ and $u^{0}$ satisfies (4). Then, solution of the linear second type Fredholm equation with weak singularity (17), is obtained from (11).

Remark 2.3. Note that the above method able us instead solving of system of equations (1), we first solve system of equations (15) and (16) and then by replacement of solutions in (17), we obtain a separate equation for $\tilde{p}$. By obtaining of $\tilde{p}$, one could obtain $p$. Also if $\lambda \in R_{\sigma}$ and $|\lambda| \longrightarrow \infty$, then $U(x-\xi, \lambda) \longrightarrow 0$. So the equation (17) could be solved easily.

Theorem 2.4. If $\lambda \in R_{\sigma}$ and $|\lambda|$ is sufficiently large, then the equation (17) has an unique smoothness solution and this solution could be obtained by the successive approximation method.

If as remark 2.3 , we have a separate equation for $\tilde{p}$ from $(17)$ and $H(x, \xi, \lambda)$ is the resolvent kernel of the equation, then

$$
H(x, \xi, \lambda)=\sum_{k=0}^{\infty}\left(\frac{-\lambda}{\nu}\right)^{k} U_{k+1}(x, \xi, \lambda),
$$


where,

$$
U_{1}(x, \xi, \lambda)=U(x-\xi, \lambda)
$$

and

$$
U_{k+1}(x, \xi, \lambda)=\int_{\mathbb{R}^{3}} U_{1}(x, \gamma, \lambda) U_{k}(\gamma, \xi, \lambda) \mathrm{d} \gamma
$$

Note that, the series (18) is uniformly convergent whenever $\lambda \in R_{\sigma},|\lambda|$ is sufficiently large and $x \neq \xi$. Also, treatments of the functions $H(x, \xi, \lambda)$ and $H(x-\xi, \lambda)$ are same ([15]). Thus,

$$
\begin{array}{r}
\tilde{p}(\xi, \lambda)= \\
=-\int_{\mathbb{R}^{3}} \sum_{i, j=1}^{3} \frac{\partial U(x-\xi, \lambda)}{\partial \xi_{i}} \mathrm{~d} x \int_{0}^{\infty} e^{-\lambda t} u_{j}(x, t) \frac{\partial u_{i}(x, t)}{\partial x_{j}} \mathrm{~d} t+ \\
\quad+\frac{\lambda}{\nu} \int_{\mathbb{R}^{3}} H(x, \xi, \lambda) d x \int_{\mathbb{R}^{3}} \sum_{p, q=1}^{3} \frac{\partial U(\gamma-x, \lambda)}{\partial \xi_{p}} \mathrm{~d} \gamma \\
\int_{0}^{\infty} e^{-\lambda t} u_{q}(\gamma, t) \frac{\partial u_{p}(\gamma, t)}{\partial \gamma_{q}} \mathrm{~d} t+\int_{\mathbb{R}^{3}} \sum_{i=1}^{3} u_{i}^{0}(x) \frac{\partial U(x-\xi, \lambda)}{\partial \xi_{i}} \mathrm{~d} x- \\
\quad-\frac{\lambda}{\nu} \int_{\mathbb{R}^{3}} H(x, \xi, \lambda) \mathrm{d} x \int_{\mathbb{R}^{3}} \sum_{p=1}^{3} u_{p}^{0}(\gamma) \frac{\partial U(\gamma-x, \lambda)}{\partial x_{p}} \mathrm{~d} \gamma .
\end{array}
$$

By using (18), (19) and (20), we have

$$
\frac{\partial U_{1}(x, \xi, \lambda)}{\partial x_{1}}=\frac{\partial U(x-\xi, \lambda)}{\partial x_{1}}=-\frac{\partial U(x-\xi, \lambda)}{\partial \xi_{1}}=-\frac{\partial U_{1}(x, \xi, \lambda)}{\partial \xi_{1}}
$$

and in general,

$$
\begin{aligned}
& \frac{\partial^{|\alpha|} U_{1}(x, \xi, \lambda)}{\partial x_{1}^{\alpha_{1}} \partial x_{2}^{\alpha_{2}} \partial x_{3}^{\alpha_{3}}}=\frac{\partial^{|\alpha|} U(x-\xi, \lambda)}{\partial x_{1}^{\alpha_{1}} \partial x_{2}^{\alpha_{2}} \partial x_{3}^{\alpha_{3}}}= \\
&=(-1)^{|\alpha|} \frac{\partial^{|\alpha|} U(x-\xi, \lambda)}{\partial \xi_{1}^{\alpha_{1}} \partial \xi_{2}^{\alpha_{2}} \partial \xi_{3}^{\alpha_{3}}}=(-1)^{|\alpha|} \frac{\partial^{|\alpha|} U_{1}(x, \xi, \lambda)}{\partial \xi_{1}^{\alpha_{1}} \partial \xi_{2}^{\alpha_{2}} \partial \xi_{3}^{\alpha_{3}}},
\end{aligned}
$$

where, $\alpha_{1}, \alpha_{2}, \alpha_{3}$ are non-negative integers and $|\alpha|=\alpha_{1}+\alpha_{2}+\alpha_{3}$. Also, 


$$
\begin{aligned}
\frac{\partial U_{2}(x, \xi, \lambda)}{\partial x_{1}} & =\frac{\partial}{\partial x_{1}} \int_{\mathbb{R}^{3}} U(x-\gamma, \lambda) U(\gamma-\xi, \lambda) \mathrm{d} \gamma= \\
& =\int_{\mathbb{R}^{3}} \frac{\partial U(x-\gamma, \lambda)}{\partial x_{1}} U(\gamma-\xi, \lambda) \mathrm{d} \gamma= \\
& =-\int_{\mathbb{R}^{3}} \frac{\partial U(x-\gamma, \lambda)}{\partial \gamma_{1}} U(\gamma-\xi, \lambda) \mathrm{d} \gamma= \\
& =\int_{\mathbb{R}^{3}} U(x-\gamma, \lambda) \frac{\partial U(\gamma-\xi, \lambda)}{\partial \gamma_{1}} \mathrm{~d} \gamma= \\
& =-\int_{\mathbb{R}^{3}} U(x-\gamma, \lambda) \frac{\partial U(\gamma-\xi, \lambda)}{\partial \xi_{1}} \mathrm{~d} \gamma= \\
& =-\frac{\partial}{\partial \xi_{1}} \int_{\mathbb{R}^{3}} U(x-\gamma, \lambda) U(\gamma-\xi, \lambda) \mathrm{d} \gamma=-\frac{\partial U_{2}(x, \xi, \lambda)}{\partial \xi_{1}}
\end{aligned}
$$

In general,

$$
\begin{gathered}
\frac{\partial^{|\alpha|} U_{2}(x, \xi, \lambda)}{\partial x_{1}^{\alpha_{1}} \partial x_{2}^{\alpha_{2}} \partial x_{3}^{\alpha_{3}}}=(-1)^{|\alpha|} \frac{\partial^{|\alpha|} U_{2}(x, \xi, \lambda)}{\partial \xi_{1}^{\alpha_{1}} \partial \xi_{2}^{\alpha_{2}} \partial \xi_{3}^{\alpha_{3}}}, \\
\frac{\partial^{|\alpha|} U_{n}(x, \xi, \lambda)}{\partial x_{1}^{\alpha_{1}} \partial x_{2}^{\alpha_{2}} \partial x_{3}^{\alpha_{3}}}=(-1)^{|\alpha|} \frac{\partial^{|\alpha|} U_{n}(x, \xi, \lambda)}{\partial \xi_{1}^{\alpha_{1}} \partial \xi_{2}^{\alpha_{2}} \partial \xi_{3}^{\alpha_{3}}}, \quad(n \geq 1),
\end{gathered}
$$

and

$$
\frac{\partial^{|\alpha|} H(x, \xi, \lambda)}{\partial x_{1}^{\alpha_{1}} \partial x_{2}^{\alpha_{2}} \partial x_{3}^{\alpha_{3}}}=(-1)^{|\alpha|} \frac{\partial^{|\alpha|} H(x, \xi, \lambda)}{\partial \xi_{1}^{\alpha_{1}} \partial \xi_{2}^{\alpha_{2}} \partial \xi_{3}^{\alpha_{3}}} .
$$

Thus, we could rewrite two last expressions in right hand side of (21) as follows

$$
\begin{gathered}
\int_{\mathbb{R}^{3}} \sum_{i=1}^{3} u_{i}^{0}(x) \frac{\partial U(x-\xi, \lambda)}{\partial \xi_{i}} \mathrm{~d} x- \\
-\frac{\lambda}{\nu} \int_{\mathbb{R}^{3}} H(x, \xi, \lambda) \mathrm{d} x \int_{\mathbb{R}^{3}} \sum_{p=1}^{3} u_{p}^{0}(\gamma) \frac{\partial U(\gamma-x, \lambda)}{\partial x_{p}} \mathrm{~d} \gamma= \\
=\int_{\mathbb{R}^{3}} \sum_{i=1}^{3} \frac{\partial u_{i}^{0}(x)}{\partial x_{i}} U(x-\xi, \lambda) \mathrm{d} x- \\
-\frac{\lambda}{\nu} \int_{\mathbb{R}^{3}} H(x, \xi, \lambda) \mathrm{d} x \int_{\mathbb{R}^{3}} \sum_{p=1}^{3} \frac{\partial u_{p}^{0}(\gamma)}{\partial \gamma_{p}} U(\gamma-x, \lambda) \mathrm{d} \gamma
\end{gathered}
$$

Note that, these two last expressions are of class $C^{\infty}$ because one could transmit to $u_{i}^{0}(x)$ all partial differentiation of the functions $U(x-\xi, \lambda)$ and 
$H(x, \xi, \lambda)$, until singularity will not appear. By replacement of (21) in (12), we find $g_{i}(x, \lambda)$ and then by replacement of the result in (15), we obtain

$$
\begin{aligned}
& \tilde{u}_{i}(\xi, \lambda)=\int_{\mathbb{R}^{3}} U(x-\xi, \lambda) \mathrm{d} x\left\{\frac{-1}{\nu} u_{i}^{0}(x)+\right. \\
& +\frac{1}{\nu} \sum_{j=1}^{3} \int_{0}^{\infty} e^{-\lambda t} u_{j}(x, t) \frac{\partial u_{i}(x, t)}{\partial x_{j}} \mathrm{~d} t+ \\
& +\frac{1}{\nu} \frac{\partial}{\partial x_{i}}\left[-\int_{\mathbb{R}^{3}} \sum_{p, q=1}^{3} \frac{\partial U(\gamma-x, \lambda)}{\partial x_{p}} \mathrm{~d} \gamma \int_{0}^{\infty} e^{-\lambda t} u_{q}(\gamma, t) \frac{\partial u_{p}(\gamma, t)}{\partial \gamma_{q}} \mathrm{~d} t+\right. \\
& +\frac{\lambda}{\nu} \int_{\mathbb{R}^{3}} H(\gamma, x, \lambda) d \gamma \int_{\mathbb{R}^{3}} \sum_{p, q=1}^{3} \frac{\partial U(\zeta-\gamma, \lambda)}{\partial \gamma_{p}} \mathrm{~d} \zeta \\
& \int_{0}^{\infty} e^{-\lambda t} u_{q}(\zeta, t) \frac{\partial u_{p}(\zeta, t)}{\partial \zeta_{q}} \mathrm{~d} t+\int_{\mathbb{R}^{3}} \sum_{p=1}^{3} u_{p}^{0}(\gamma) \frac{\partial U(\gamma-x, \lambda)}{\partial x_{p}} \mathrm{~d} \gamma- \\
& \left.\left.-\frac{\lambda}{\nu} \int_{\mathbb{R}^{3}} H(\gamma, x, \lambda) \mathrm{d} \gamma \int_{\mathbb{R}^{3}} \sum_{p=1}^{3} u_{p}^{0}(\zeta) \frac{\partial U(\zeta-\gamma, \lambda)}{\partial \gamma_{p}} \mathrm{~d} \zeta\right]\right\}= \\
& =\frac{-1}{\nu} \int_{\mathbb{R}^{3}} u_{i}^{0}(x) U(x-\xi, \lambda) \mathrm{d} x+ \\
& +\frac{1}{\nu} \sum_{j=1}^{3} \int_{\mathbb{R}^{3}} U(x-\xi, \lambda) \mathrm{d} x \int_{0}^{\infty} e^{-\lambda t} u_{j}(x, t) \frac{\partial u_{i}(x, t)}{\partial x_{j}} \mathrm{~d} t- \\
& -\frac{1}{\nu} \int_{\mathbb{R}^{3}} U(x-\xi, \lambda) \mathrm{d} x \int_{\mathbb{R}^{3}} \sum_{p, q=1}^{3} \frac{\partial U(\gamma-x, \lambda)}{\partial x_{i}} \mathrm{~d} \gamma \\
& \int_{0}^{\infty} e^{-\lambda t} \frac{\partial u_{q}(\gamma, t)}{\partial \gamma_{p}} \frac{\partial u_{p}(\gamma, t)}{\partial \gamma_{q}} \mathrm{~d} t+\frac{\lambda}{\nu^{2}} \int_{\mathbb{R}^{3}} U(x-\xi, \lambda) \mathrm{d} x \\
& \int_{\mathbb{R}^{3}} \frac{\partial H(\gamma, x, \lambda)}{\partial x_{i}} \mathrm{~d} \gamma \int_{\mathbb{R}^{3}} \sum_{p, q=1}^{3} U(\zeta-\gamma, \lambda) \mathrm{d} \zeta \\
& \int_{0}^{\infty} e^{-\lambda t} \frac{\partial u_{q}(\zeta, \lambda)}{\partial \zeta_{p}} \frac{\partial u_{p}(\zeta, t)}{\partial \zeta_{q}} \mathrm{~d} t, \quad\left(i=1,2,3 ; \xi \in \mathbb{R}^{3}\right) .
\end{aligned}
$$

Note that right hand side of expression of above equation is of class $C^{\infty}$. Now for each $n \geq 0$, put

$$
u_{m}^{(n)}(\xi, t)=\frac{1}{2 \pi \sqrt{-1}} \int_{c-\sqrt{-1} \infty}^{c+\sqrt{-1} \infty} e^{\lambda t} \tilde{u}_{m}^{(n)}(\xi, \lambda) \mathrm{d} \lambda,
$$


where, $m=1,2,3, c>0, \xi \in \mathbb{R}^{3}, u_{m}^{(0)}=u_{m}^{0},(n)$ is only a symbol and

$$
\begin{aligned}
& \tilde{u}_{m}^{(n)}(\xi, \lambda)=\frac{-1}{\nu} \int_{\mathbb{R}^{3}} U(x-\xi, \lambda) u_{m}^{0}(x) \mathrm{d} x+ \\
& +\frac{1}{\nu} \sum_{j=1}^{3} \int_{\mathbb{R}^{3}} U(x-\xi, \lambda) \mathrm{d} x \int_{0}^{\infty} e^{-\lambda t} u_{j}^{(n-1)}(x, t) \frac{\partial u_{m}^{(n-1)}(x, t)}{\partial x_{j}} \mathrm{~d} t-
\end{aligned}
$$

$$
\begin{gathered}
-\frac{1}{\nu} \int_{\mathbb{R}^{3}} U(x-\xi, \lambda) \mathrm{d} x \int_{\mathbb{R}^{3}} \sum_{p, q=1}^{3} \frac{\partial U(\gamma-x, \lambda)}{\partial x_{m}} \mathrm{~d} \gamma \\
\int_{0}^{\infty} e^{-\lambda t} \frac{\partial u_{q}^{(n-1)}(\gamma, t)}{\partial \gamma_{p}} \frac{\partial u_{p}^{(n-1)}(\gamma, t)}{\partial \gamma_{q}} \mathrm{~d} t+ \\
+\frac{\lambda}{\nu^{2}} \int_{\mathbb{R}^{3}} U(x-\xi, \lambda) \mathrm{d} x \int_{\mathbb{R}^{3}} \frac{\partial H(\gamma, x, \lambda)}{\partial x_{m}} \mathrm{~d} \gamma \\
\int_{\mathbb{R}^{3}} \sum_{p, q=1}^{3} U(\zeta-\gamma, \lambda) \mathrm{d} \zeta \int_{0}^{\infty} e^{-\lambda t} \frac{\partial u_{q}^{(n-1)}(\zeta, \lambda)}{\partial \zeta_{p}} \frac{\partial u_{p}^{(n-1)}(\zeta, t)}{\partial \zeta_{q}} \mathrm{~d} t
\end{gathered}
$$

where $m=1,2,3, n \geq 1, \xi \in \mathbb{R}^{3}$ and $\lambda \in R_{\sigma}$.

If $u_{m}^{(n-1)}(x, t)$ satisfies (6) and (7), for all $n \geq 1$ and $m=1,2,3$, then $u_{m}^{(n)}(x, t)$ so is for all $n \geq 1$, because $\lambda \in R_{\sigma}$. Also by $(25), u_{m}^{(n)}(\xi, t)$ satisfies (6) and (7), for all $n \geq 1$ and $m=1,2$, 3. Since, every $\tilde{u}_{m}^{(n)}(\xi, \lambda)$ is of class $C^{\infty}$ respect to $\xi$, so is $u_{m}^{(n)}(\xi, t)$. On the other hand, every $\tilde{u}_{m}^{(n)}(\xi, \lambda)$ is sufficiently small when $\lambda \in R_{\sigma}$ and $|\lambda| \longrightarrow \infty$, so $u_{m}^{(n)}(\xi, t)$ is of class $C^{\infty}$ for all $n \geq 1$ and $m=1,2,3$.

Remark 2.5. For arbitrary $d>0$, put

$$
\begin{gathered}
\Gamma=\{c+i t:-d \leq t \leq d\} \bigcup\left\{\lambda_{1}+i \lambda_{2}: \lambda_{2}+d=\operatorname{tg} \delta\left(\lambda_{1}-c\right)\right\} \\
\bigcup\left\{\lambda_{1}+i \lambda_{2}: \lambda_{2}-d=-\operatorname{tg} \delta\left(\lambda_{1}-c\right)\right\} .
\end{gathered}
$$

Then, by Cauchy Theorem ([15])

$$
\int_{c-\sqrt{-1} \infty}^{c+\sqrt{-1} \infty} e^{\lambda t} \tilde{u}_{m}^{(n)}(\xi, \lambda) \mathrm{d} \lambda=\int_{\Gamma} e^{\lambda t} \tilde{u}_{m}^{(n)}(\xi, \lambda) \mathrm{d} \lambda .
$$


Since $\operatorname{Re}(\lambda) \longrightarrow-\infty$ in the right integral, the left integral exists. For $1 \leq m \leq 3$, define

$$
\begin{gathered}
T_{m}\left(\tilde{u}^{(n-1)}(\xi, \lambda)\right)=\frac{1}{2 \pi \sqrt{-1}} \int_{c-\sqrt{-1} \infty}^{c+\sqrt{-1} \infty} e^{\lambda t}\left\{\frac{1}{\nu} \sum_{j=1}^{3} \int_{\mathbb{R}^{3}} U(x-\xi, \lambda) \mathrm{d} x\right. \\
\int_{0}^{\infty} e^{-\lambda t} u_{j}^{(n-1)}(x, t) \frac{\partial u_{m}^{(n-1)}(x, t)}{\partial x_{j}} \mathrm{~d} t- \\
-\frac{1}{\nu} \int_{\mathbb{R}^{3}} U(x-\xi, \lambda) \mathrm{d} x \int_{\mathbb{R}^{3}} \sum_{p, q=1}^{3} \frac{\partial U(\gamma-x, \lambda)}{\partial x_{m}} \mathrm{~d} \gamma \\
\int_{0}^{\infty} e^{-\lambda t} \frac{\partial u_{q}^{(n-1)}(\gamma, t)}{\partial \gamma_{p}} \frac{\partial u_{p}^{(n-1)}(\gamma, t)}{\partial \gamma_{q}} \mathrm{~d} t+ \\
+\frac{1}{\nu^{2}} \int_{\mathbb{R}^{3}} U(x-\xi, \lambda) \mathrm{d} x \int_{\mathbb{R}^{3}} \frac{\partial H(\gamma, x, \lambda)}{\partial x_{m}} \mathrm{~d} \gamma \\
\left.\int_{\mathbb{R}^{3}} \sum_{p, q=1}^{3} U(\zeta-\gamma, \lambda) \mathrm{d} \zeta \int_{0}^{\infty} e^{-\lambda t} \frac{\partial u_{q}^{(n-1)}(\zeta, \lambda)}{\partial \zeta_{p}} \frac{\partial u_{p}^{(n-1)}(\zeta, t)}{\partial \zeta_{q}} \mathrm{~d} t\right\} .
\end{gathered}
$$

Then, by (25)

$$
u_{m}^{(n)}(\xi, t)=T_{m}\left(u^{(n-1)}(\xi, \lambda)\right)+h_{m}(\xi, t)
$$

where,

$$
h_{m}(\xi, t)=\frac{1}{2 \pi \sqrt{-1}} \int_{c-\sqrt{-1} \infty}^{c+\sqrt{-1} \infty} e^{\lambda t}\left\{\frac{-1}{\nu} \int_{\mathbb{R}^{3}} U(x-\xi, \lambda) u_{m}^{0}(x) \mathrm{d} x\right\} .
$$

Finally, suppose that $S$ is the set of all functions satisfy (6) and (7).

Theorem 2.6. The transformation $T_{m}: S \longrightarrow S$ is a contraction and the sequence $\left\{u_{m}^{(n)}\right\}_{n \geq 1}$ is convergent for all $m=1,2,3$. If $u_{m}^{(n)} \longrightarrow u_{m}$ $(m=1,2,3)$, then $u=\left(u_{1}, u_{2}, u_{3}\right)$ is the unique solution of the problem $A$.

Now, if we put in $(21)$ these $u_{1}, u_{2}$ and $u_{3}$, we could find $\tilde{p}(\xi, \lambda)$ and so we could find

$$
p(x, t)=\frac{1}{2 \pi \sqrt{-1}} \int_{c-\sqrt{-1} \infty}^{c+\sqrt{-1} \infty} e^{\lambda t} \tilde{p}(x, \lambda) \mathrm{d} \lambda .
$$

Therefore, the problem A be solved completely.

\section{REFERENCES}

[1] N. Aliev, M. Jahanshahi, Sufficient Conditions for Reduction of the BVP Including a Mixed PDE with Non-local Boundary Conditions to Fredholm Integral Equations, Int. J. Math. Educ. Sci. Technol., Vol. 28, No. 3 (1997), 419-425. 
[2] N. Aliev, S.M. Hosseini, An Analysis of a Parabolic Problem with General (Non-local and Global) Supplementary Linear Conditions I, Italian J. of Pure and Appl. Math., No. 12 (2002), 143-154.

[3] N. Aliev, S.M. Hosseini, An Analysis of a Parabolic Problem with General (Non-local and Global) Supplementary Linear Conditions II, Italian J. of Pure and Appl. Math., No. 13 (2003), 115-127.

[4] N. Aliev, Sh. Rezapour, M. Jahanshahi, On Fefferman's Non-existence Problems, Mathematica Morvica, Vol. 11 (2007), 1-7.

[5] V.I. Arnold, Ordinary Differential Equations, Springer-Verlag, 1992.

[6] A.V. Bitsadze, Boundary Value Problems including Second Order Elliptic Equations, North-Holland Publication, First edition, 1968.

[7] R. Courant, D. Hilbert, Methods of Mathematical Physics, Vol. II, John-Wiely, 1953.

[8] A.A. Dezin, Partial Differential Equations, Springer-Verlag, 1987.

[9] A.A. Dorodnichen, On the Approach to the Solution of Boundary Value Problems for Differential Equations (Computational Experiment), Contemporary Problems of Applied Mathematics and Mathematical Physics, Moscow, 1988, 31-42 (in Russian).

[10] S. M. Hosseini, N. Aliev, A Mixed Parabolic with a Non-local and Global Linear Conditions, J. Sci. I. R. Iran, Vol. 11, No. 3 (2000), 233-237.

[11] S. M. Hosseini, N. Aliev, Sufficient Conditions for the Reduction of the BVP for PDE with Non-local and Global Boundary Conditions to Fredholm Integral Equations (on a Rectangular Domain, Appl. Math. and Computation, 147 (2004), 669-685.

[12] G. Kavei, N. Aliev, An Analytical Methods to the Solution of the Time-dependent Schrodinger Equation Using Half Cylinder Space System-I, Bull. of Pure and Appl. Sciences, Vol. 16E, No. 2 (1997), 253-263.

[13] G. Kavei, N. Aliev, The Existence and Uniqueness of the Solution of the Spectral Problem II, J. Sci. I.R. Iran, Vol. 10, No. 4 (1999), 252-258.

[14] A.K. Pani, G. Fairweather, $H^{1}$-Galerkin Mixed Finite Element Methods for Parabolic Partial Integro-differential Equations, IMA J. Numer. Anal., 22, no. 2 (2002), 231252.

[15] M.L. Rasulov, Methods of Contour Inegration, North-Holland, 1967.

[16] S.G. Samko, A.A. Kilbas, O.I. Marichev, Integrals and Derivatives of Fractional Order and Some of their Applications, (in Russian), Nauka and Technika, Minsk, 1987.

[17] S. Shahmorad, Numerical Solution of the General Form linear Fredholm-Volterra Integro-differential Equational Equations by the Tau Method with an Error Esimation, to appear in Appl. Math. and Computation (2004).

[18] V.S. Vladimirov, Equations of Mathematical Physics, Mir Publisher, Moscow, 1984.

[19] V.S. Vladimirov, Generalized Functions in Mathematical Physics, Mir Publisher, Moscow, 1979. 
[20] V. Volterra, Theorie Mathematique de la Lutte Pour la Vie, Paris, 1931.

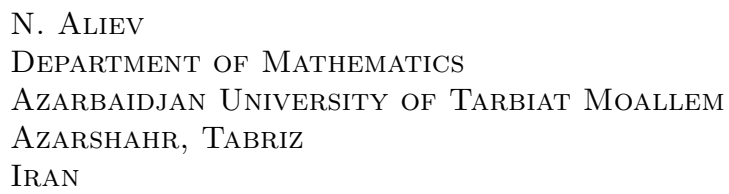

Sh. ReZApour

Department of Mathematics

Azarbaidjan University of Tarbiat Moallem Azarshahr, TABRIZ

IRAN

E-mail address: sh.rezapour@azaruniv.edu

M. JAHANSHAHI

Department of Mathematics

Azarbaidjan University of Tarbiat Moallem AZARSHAHR, TABRIZ

IRAN

E-mail address: jahan_m15@yahoo.com 